\title{
Study on Tribological Property of PMMA Denture Base Materials with Negative Ion Powder
}

\section{Mei-tian LIU ${ }^{1,2, *}$, Xiao-hui ZHANG ${ }^{1,2}$, Jing-ting ZHANG ${ }^{1,2}$, Qian ZHENG ${ }^{1,2}$ and Bin LIU ${ }^{3}$}

\author{
${ }^{1}$ Key Laboratory of Oral Diseases of Gansu Province, Northwest University for Nationalities, \\ Lanzhou 730030, P. R. China \\ ${ }^{2}$ Key Laboratory of Stomatology of State Ethnic Affairs Commission, Northwest University for \\ Nationalities, Lanzhou 730030, P. R. China \\ ${ }^{3}$ College of Stomatology of Lanzhou University, Lanzhou 730000, P.R. China \\ *liumt2009@163.com
}

Keywords: Polymethylmethacrylate, Anion powder, Denture, Tribological properties.

\begin{abstract}
Objective: To prepare the denture base material with anion-containing denture, and to test the friction properties of the denture base materials with different mass percentage of negative ion. Methods: Negative ion powders were added into the denture base materials by ball milling blending at $0 \%, 1 \%, 2 \%, 3 \%, 4 \%$ and $5 \%$. According to oral denture base production process, the standard samples $(19 \mathrm{~mm} \times 10 \mathrm{~mm} \times 4 \mathrm{~mm})$ were prepared and numbered. The experiment was divided into dry friction group and artificial saliva wet friction group. Each test group was tested for three times, and the mean value was calculated. Using scanning electron microscope (SEM) to observe the surface scratches of $0 \% \mathrm{wt}, 2 \% \mathrm{wt}, 5 \% \mathrm{wt}$ samples. Results: Under the condition of dry friction, the volume wear rate decreased with the increase of the content of negative ion powder, the friction coefficient did not change significantly compared with the untreated group, the volume wear rate decreased under the condition of artificial saliva lubrication. The friction coefficient also did not change significantly. Compared with the untreated group, the SEM results showed that under the condition of dry grinding, the furrows and cracks on the surface of the sample were less, while under the artificial saliva lubrication condition, the surface of the specimen was smoother. Conclusion: The denture base material containing more than $2 \%$ wt of anion powder has good tribological properties.
\end{abstract}

\section{Introduction}

Poly (methyl methacrylate) has been widely used in the manufacture of oral denture base. It is the most widely used denture base material because of its simple operation, easy repairing and good biocompatibility. At the same time, because of its poor wear resistance, prone to aging and so on, so that its clinical life is greatly reduced. In clinical applications, denture base surface or resin teeth surface has serious wear marks. Resin tooth surface was flat, after such problems, attracted many thoughts of scholars. A large number of literature [1-4] proved that the addition of inorganic nanoparticles can improve the tribological properties of PMMA. A. Akinci et. al [1] successfully prepared zirconia-containing PMMA materials, the results suggest: zirconia enhanced PMMA friction and wear properties. The surface treatment of titanium dioxide can enhance the wear resistance of PMMA composites, and the surface treatment of titanium dioxide is effective. At the same time, it was found that the mechanical properties of PMMA denture base material with negative ion powder prepared by adding anion powder into PMMA denture powder were improved within a certain range [2]. So we tried to explore the effect of different content of negative ion powder on the influence of PMMA tribological properties. 


\section{Experimental Section}

\section{Experimental Materials and Equipment}

Experimental materials

\begin{tabular}{|c|c|}
\hline Name & Factory \\
\hline Anion powder & $\begin{array}{l}\text { Ultra-fine powder from the processing of the } \\
\text { laboratory. Shijiazhuang. Hebei }\end{array}$ \\
\hline Anhydrous ethanol & $\begin{array}{l}\text { (Tianjin) Pharmaceutical and Chemical } \\
\text { Company Limited }\end{array}$ \\
\hline $\begin{array}{l}\text { Glycidyl methacrylate(GMA) } \\
\text { N, N-dimethylformamide(DMF) }\end{array}$ & $\begin{array}{l}\text { Shiphan Chemical(Shanghai)Co., Ltd } \\
\text { (Tianjin) Pharmaceutical and Chemical } \\
\text { Company Limited }\end{array}$ \\
\hline $\begin{array}{l}\text { High - purity deionized water(DW) } \\
\text { Fused denture base powder and water liquid } \\
\text { Silicon nitride ceramic ball }\end{array}$ & $\begin{array}{l}\text { Millipore, USA } \\
\text { Dentsply Dental Corporation } \\
\text { Shanghai Xinmao Precision Ceramic } \\
\text { Technology Co., Ltd }\end{array}$ \\
\hline
\end{tabular}

Experimental equipment

\begin{tabular}{ll}
\hline Name & Factory \\
\hline Laser dynamic particle size analyzer & Microtrac S3500 Particle Size Analyzer, \\
& Microtrac Inc., PA \\
X - ray diffraction & D/max-2400, Japan, Rigaku \\
Scanning electron microscope & SEM, JEOL, 5600LV, Japan \\
Electric heating constant temperature drying & The earth Automatic Instrument Factory, \\
box & Jintan City, Jiangsu Province \\
Electric heating constant temperature air & DHG-9035A, Shanghai Yi Heng \\
blast drying box & Experimental Instrument Co., Ltd. \\
Heat collection type constant temperature & Gongyi yingyu Yuhua Instrument Factory \\
heating magnetic agitator & Optima L-100XP, USA \\
Speed centrifuge & Ulverisette 7, FRITSCH Co.,LTD.Germany \\
Micro planetary high-energy ball mill & Guangzhou, China \\
WDW-200 Universal material testing & Hengyi precision instrument Co. LTD.China \\
machine & SEM JSM,5600LV,Japan \\
Micro hardness tester & CETR Limited company, USA \\
Low vacuum scanning electron microscope & SW-CJ-IFD, China, J iangsu \\
Reciprocating friction tester & Shanghai, CHINA Miaojie \\
Super clean working table & Laizhou city Wei instrument test equipment \\
Stainless steel steam sterilizing device & manufacturing Co., Ltd. \\
Metallographic sample polishing machine & BRANSON 200 \\
Ultrasonic cleaner & ATR-FTIR,IFS66V/S, Bruker \\
Total reflection Fourier transform infrared & \\
spectroscopy & \\
\hline
\end{tabular}

\section{Anion Powder Ultrafine Treatment}

$15 \mathrm{~g}$ of anion powder was weighed and placed in a ball mill. $100 \mathrm{ml}$ of anhydrous ethanol (analytical reagent, Tianjin Reagent Co., Ltd.) was added, ultrasonic dispersion was performed for $1 \mathrm{~h}$, high speed ball milling for $4 \mathrm{~h}, 8 \mathrm{~h}, 12 \mathrm{~h}, 16 \mathrm{~h}$ and $22 \mathrm{~h}$. And then ultrasonic dispersion 30min, drying, grinding, screening, numbering .

\section{Anion Powder Surface Treatment}

$60 \mathrm{ml}$ of $\mathrm{N}, \mathrm{N}$-dimethylformamide (DMF), $3 \mathrm{~g}$ of anion powder and $1.13 \mathrm{~g}$ of GMA were placed in a $100 \mathrm{ml}$ three-necked flask after ultrasonic dispersion for 1 hour. The mixture was heated to 98$100{ }^{\circ} \mathrm{C}$ in an oil bath. After $10 \mathrm{~h}$ of vigorous stirring, centrifugation $(7500 \mathrm{r} / \mathrm{min}, 15 \mathrm{~min})$, to wash the ungrafted material and the inhibitor for 5 times with ultra-pure water. Pre-drying $\left(50{ }^{\circ} \mathrm{C}, 4 \mathrm{~h}\right)$, vacuum drying $\left(50{ }^{\circ} \mathrm{C}, 24 \mathrm{~h}\right)$, grinding, sieving and reserved for use later. 


\section{Sample Preparation Method}

The non-treated and refined negative ion powders were added into the denture base material by ball-milling blending method, respectively, with $0 \%, 1 \%, 2 \%, 3 \%, 4 \%$ and $5 \%$ speed $900 \mathrm{rpm}$, time $1 \mathrm{~h}$. According to the denture base manufacturing process, the composite denture powder and denture water are mixed according to the ratio of 2: 1 (weight ratio), and evenly mixed with a stainless steel knife and gently shaken. When the dough period is filled in the plaster model, polyethylene film on the rear cover type box, slowly pressurized open type box, peeled off the polyethylene film, remove the excess material around, and then covered with lid and pressed in the box press. Put in cold water to boil, turn off the heat for 30 minutes, cook until boiling, boiling water for 20 minutes in the continued, cooled to room temperature, open the box, remove the embryo-like, with 500,600,800 water sandpaper grinding and polishing, so that the upper and lower surface parallel and smooth. Using the above methods, were prepared $19 \mathrm{~mm} \times 10 \mathrm{~mm} \times 4 \mathrm{~mm}$ standard sample and numbered them.

\section{Test Methods}

The main composition and proportion of artificial saliva were shown in Table 1 ( $0 \% \mathrm{wt}, 2 \% \mathrm{wt}$, 5\%wt). The experimental conditions were as follows: experimental group: artificial friction group and artificial saliva [5-7], $\mathrm{F}=10 \mathrm{~N}$ (normal chewing bite force in the mouth at $3 \sim 36 \mathrm{~N}$ ), $\mathrm{T}=60$ $\min , A=5 \mathrm{~mm}, \mathrm{f}=2 \mathrm{~Hz}$. The sample size is: $19 \mathrm{~mm} \times 10 \mathrm{~mm} \times 4 \mathrm{~mm}$, dual: $\Phi=3 \mathrm{~mm}$. The samples were washed by ultrasonic for 30 min before each friction test. Each test was replaced with a new ceramic ball or a new contact surface with ceramic balls. Each test group was tested three times. And the average thereof was calculated. Friction coefficient formula:

$$
\mathrm{M}=\mathrm{F} / \mathrm{N}
$$

M-friction coefficient, F-friction force (unit: N), N-normal load force (unit: N)

Volume wear rate of the formula:

$$
\mathrm{K}=\mathrm{V} / \mathrm{LN}
$$

(Unit: $\mathrm{mm} 3$ ), L: total sliding distance (unit: $\mathrm{mm}$ ), N: normal phase load (unit: $\mathrm{N}$ ), K-attrition rate (unit: $\mathrm{mm} 3$ / Nm), V-attrition volume.

Table 1 Composition of artificial saliva and dynamic viscosity and density at $36{ }^{\circ} \mathrm{C}$ [5-7].

\begin{tabular}{ll}
\hline Composition & Value \\
\hline $\mathrm{NaCl}(\mathrm{g})$ & 0.4 \\
$\mathrm{KCl}(\mathrm{g})$ & 0.4 \\
$\mathrm{CaCl}_{2} \cdot 2 \mathrm{H}_{2} \mathrm{O}(\mathrm{g})$ & 0.795 \\
$\mathrm{NaH}_{2} \mathrm{PO}_{4} \cdot 2 \mathrm{H}_{2} \mathrm{O}$ & 0.78 \\
$(\mathrm{~g})$ & 0.005 \\
$\mathrm{Na}_{2} \mathrm{~S} \cdot 9 \mathrm{H}_{2} \mathrm{O}(\mathrm{g})$ & 1 \\
$\mathrm{Uraa}(\mathrm{g})$ & 1000 \\
Distilled water & $0.671 \pm 0.00$ \\
$(\mathrm{~mL})$ & 1 \\
Viscosity $(\mathrm{cP})$ & $0.996 \pm 0.00$ \\
Density $(\mathrm{g} / \mathrm{mL})$ & 2 \\
\hline
\end{tabular}




\section{Results and Discussion}

PMMA Denture Base Material Containing Anion Powder in the Artificial Saliva Lubrication of the Friction Coefficient

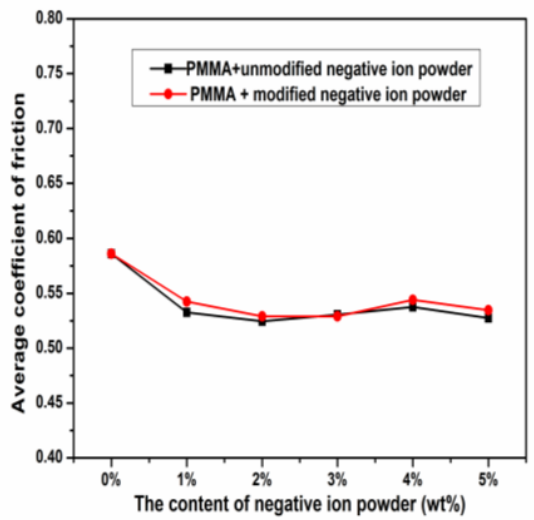

Fig. 1 The change of the friction coefficient of PMMA denture containing negative ion powder under the condition of dry grinding.

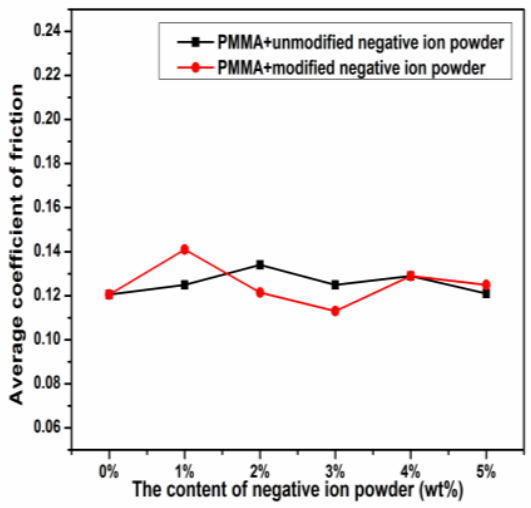

Fig. 2 The change of the friction coefficient of PMMA denture containing negative ion powder under the condition of lubrication of artificial saliva.

Wear Rate of PMMA Denture Base Material Containing Anion Powder in Artificial Saliva Lubricated State

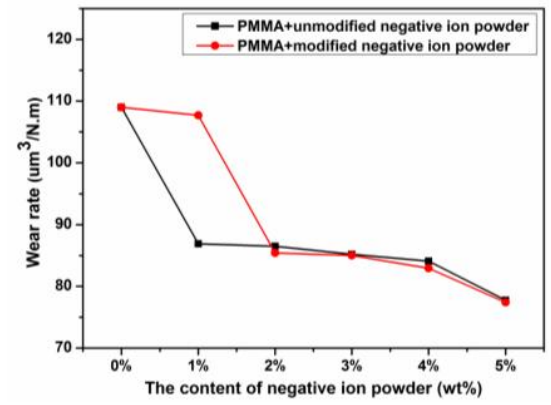

Fig. 3 The change of The volume wear rate of PMMA denture containing negative ion powder. 


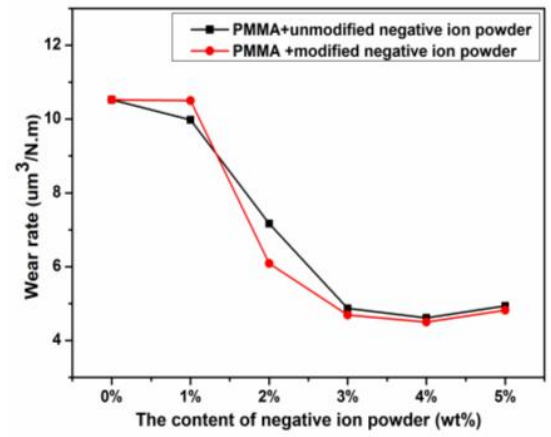

Fig. 4 The change of The volume wear rate of PMMA denture containing negative ion powder under the condition of lubrication of artificial saliva.

\section{Surface Morphology of PMMA Denture Base Material Containing Negative Ion Powder in Artificial Saliva Lubricated State}
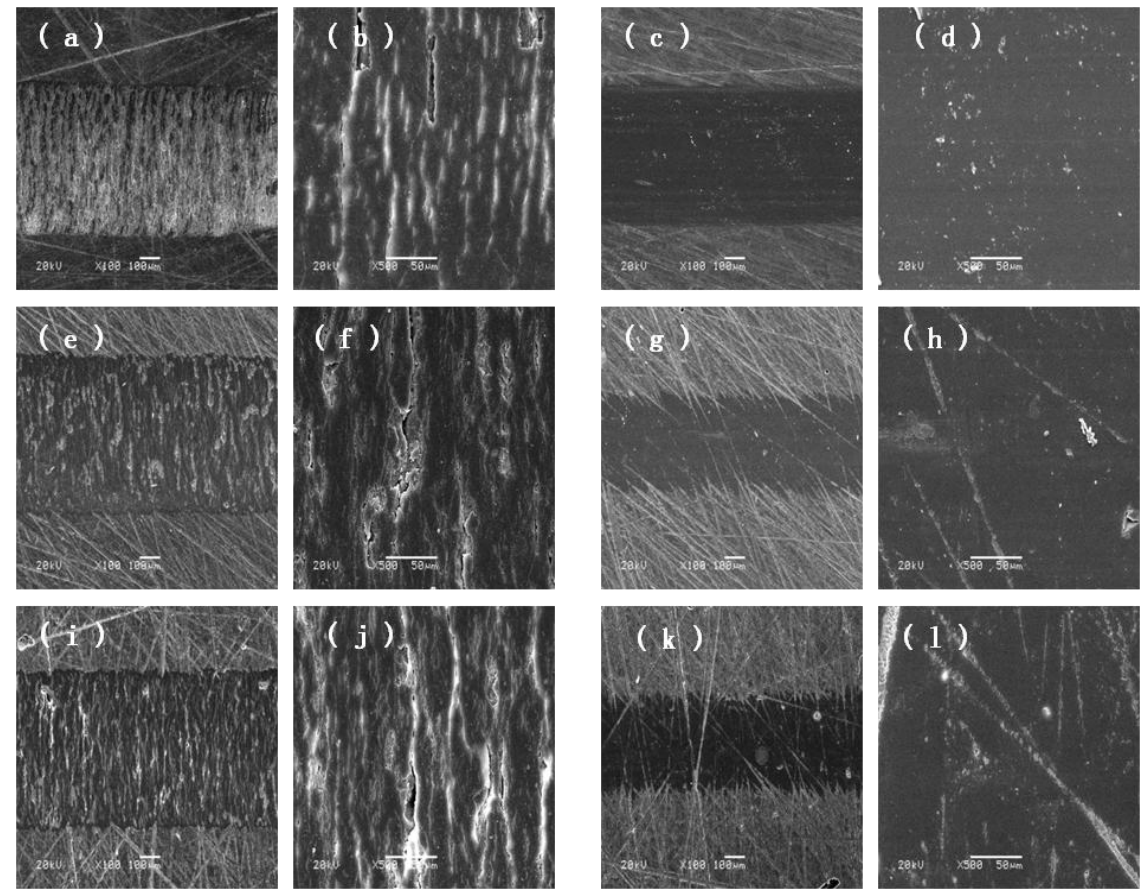

Fig. 5 The surface wear morphology characteristics of denture base composite material.

(0\%wt:a,b,c,d;2\%wt:e,f,g,h;5\%wt:I,j,k,lunder the condition of dry grinding: a, b, e, f, i, j; under the condition of lubrication of artificial saliva: c, d, g, h, k, l)

Nano-inorganic particles added to the polymer, the purpose was to enhance its friction properties. In recent years, many nanoparticles, such as silicon [8, 9], zinc oxide [10], have been added into the resin material to try to improve the tribological properties of resin materials. It can be seen from Fig. 1 and Fig. 2 that the friction coefficient of the treated and untreated groups was almost stable under dry friction conditions. In the artificial saliva lubrication environment, compared with the untreated group, the friction coefficient of the treated group fluctuated a little. The main reason was related to the quantity of saliva and heat production during the process of experiment. There was also an important reason for the control of the conditions in the preparation of the experimental sample. But in general, the friction coefficient of the composites did not fluctuate greatly under the condition of dry friction or artificial saliva lubrication, and was basically stable. It can be seen from Figure 3 and Figure 4: with the increase of negative ion powder, the volume of the overall wear rate showed a downward trend. As it can be seen in Fig. 3, the volume wear rate of the $2 \%$ treatment group was significantly reduced by $24 \mathrm{um} 3 / \mathrm{N} \cdot \mathrm{m}$ compared with $1 \%$ of the treated sample under dry friction conditions. In Fig. 4, in the artificial saliva lubrication condition, the volume wear rate of $2 \%$ 
samples decreased by $5 \mathrm{um} 3 / \mathrm{N} \cdot \mathrm{m}$ compared with the control and $1 \%$ samples. The volume wear rate decreased slowly of $3 \%$ group compared to $2 \%$ group. The volume wear rate of $3 \%, 4 \%, 5 \%$ treatment groups and the same proportion of the volume of untreated group was basically stable. It can be seen from Fig. 5 that under the condition of dry friction (a, b, e, f, i, j), there were furrows and fissures on the surface of the worn surface, but the depth of furrow was different and the number of fissures was different. Compared with the control group, under the 500 times microscope, the furrow is the shallowest, the smallest number of cracks is $\mathrm{j}$, that is, 5\% of the sample. In the artificial saliva lubrication conditions, the experimental group of the sample surface is smoother than the control sample surface. As it can be seen from Figure d, the control group surface has obvious scratches, and friction debris, but the graph L surface scratches was significantly lighter, and no friction debris. To some extent, hard particles contributed to the development of matrix toughness and hardness. Surface treatment of negative ion powder plays a significant role in the friction process. It was analyzed that the mechanism of friction changed from adhesive wear to fatigue wear. Added GMA-treated anion powder into the denture base material could enhance the internal adhesion. It was found from the hardness test in the previous mechanical properties that the addition of anion powder could increase the hardness of the surface, and we can try to explain the cause of the phenomenon of the friction surface topography from this aspect.

\section{Conclusion}

In the dry friction environment, the volume wear rate decreased with the increasing content of negative ion powder $(0 \% \mathrm{wt}, 2 \% \mathrm{wt}, 5 \% \mathrm{wt})$, the friction coefficient did not change significantly compared with the untreated group. In the artificial saliva lubrication environment, the volume wear rate decreased, there was no significant change in the coefficient of friction. Compared with the untreated group, SEM showed that under the condition of dry grinding, the furrows and cracks on the surface of the sample were less, but under the artificial saliva lubrication condition, the surface of the specimen was smoother. So the addition of anion powder could enhance the tribological properties of PMMA.

\section{Acknowledgments}

This work was supported by 1.The Fundamental Research Funds for the Central Universities, the project number: 3192013003, 2. Gansu Provincial Natural Science Foundation, the fund number: 1506RJZA279, 3. Northwest University for Nationalities Research Innovation Team (xbmucxtd2011-1).

\section{References}

[1] A. Akinci, S. Sen, U. Sen, Friction and wear behavior of zirconium oxide reinforced PMMA composites, Compos. Part B: Eng. 56: 42-47 (2014).

[2] S.O. Alsharif, H.B. Akil, N.A. Abd El-Aziz, Z.A. Bin Ahmad, Effect of alumina particles loading on the mechanical properties of light-cured dental resin composites, Mater. Des. 54: 430435 (2014).

[3] Y. Hu, G. Gu, S. Zhou, L. Wu, Preparation and properties of transparent PMMA/ZrO2 nanocomposites using 2-hydroxyethyl methacrylate as a coupling agent, Polymer 52: 122-129 (2012).

[4] M.L. Saladino, T.E. Motaung, A.S. Luyt, A. Spinella, G. Nasillo, E. Caponetti, The effect of silica nanoparticles on the morphology, mechanical properties and thermal degradation kinetics of PMMA, Polym. Degrad. Stab. 97: 452-459 (2012). 
[5] J. Zheng, Z.R. Zhou, J. Zhang, H. Li, H.Y. Yu, On the friction and wear behaviour of human tooth enamel and dentin, Wear. 255 (7-12): 967-974 (2003).

[6] H. Li, Z.R. Zhou, Wear behaviour of human teeth in dry and artificial saliva conditions, Wear. 249 (10-11): 980-984 (2001).

[7] H.Y. Yu, Z.B. Cai., P.D. Ren, M.H. Zhu, Z.R. Zhou, Friction and wear behavior of dental feldspathic porcelain, Wear. 261(5-6): 611-621 (2006).

[8] H.J. Zhou, M.Z. Rong, M.Q. Zhang, K. Friedrich, Effects of reactive compatibilization on the performance of nano-silica filled polypropylene composites, J. Mater Sci. 41: 5767-5770 (2006).

[9] X.R. Zhang, X.Q. Pei, Q.H. Wang, Friction and wear studies of polyimide composites filled with short carbon fibers and graphite and micro SiO2, Mater Des. 30: 4414-4420 (2009).

[10]N.L. McCook, B. Boesl, D.L. Burris, W.G. Sawyer, Epoxy, ZnO, and PTFE nanocomposite: friction and wear optimization, Tribol Lett. 22: 253-257 (2006). 